\title{
Remoción de atenuación y dispersión en registros preapilados mediante filtro inverso con factor de calidad estimado en espectro instantáneo
}

\author{
Álvaro Corrales ${ }^{1}$, Ovidio Almanza ${ }^{2}$, Luis Montes-Vides ${ }^{3, *}$ \\ ${ }^{1}$ Occidental de Colombia, Inc., Bogotá, Colombia \\ ${ }^{2}$ Universidad Nacional de Colombia, Departamento de Física, Bogotá, Colombia \\ ${ }^{3}$ Universidad Nacional de Colombia, Departamento de Geociencias, Bogotá, Colombia
}

\begin{abstract}
Resumen
La atenuación diferencial de los armónicos de mayor frecuencia y la dispersión asociada a las distintas velocidades de fase deterioran la imagen sísmica del subsuelo y dificultan su interpretación e inversión. Para remover la atenuación y la dispersión de los registros sísmicos, y así mejorar la imagen del subsuelo, es necesario usar filtros inversos basados en modelos que incluyen el factor de calidad $Q$, por lo que la estimación de su valor requiere un método sólido que suministre valores confiables. La descomposición adaptativa en tiempo y frecuencia (Matching Pursuit Decomposition, MPD) genera un espectro de frecuencia para cada muestra de la traza (instantánea), donde se estiman valores de $Q$ confiables, sin los inconvenientes propios de los análisis espectrales con ventanas basados en las transformadas de Fourier y de Gabor. Dado que el componente de fase del filtro es intrínsecamente estable, la compensación se aplica solo a la amplitud. Se desarrollaron algoritmos en MATLAB, los cuales implementan la técnica MPD, así como un filtro inverso estable para remover los efectos de la atenuación y la dispersión de los registros sísmicos preapilados y postapilados. La estimación del valor de $Q$ en sismogramas sintéticos y su uso con el filtro inverso permitieron corregir la distorsión de la ondícula mediante la recuperación de los espectros de amplitud y de fase. La metodología se aplicó en registros preapilados de una línea sísmica marina PSTM, incrementando la relación entre señal y ruido y mejorando ostensiblemente la resolución temporal.
\end{abstract}

Palabras clave: atenuación, dispersión, espectro instantáneo, descomposición adaptable, filtro inverso, factor de calidad.

Removal of attenuation and dispersion in pre-stack gathers using inverse filter with quality factor estimated on instantaneous spectrum

\begin{abstract}
Physical phenomena such as attenuation of high frequency components and velocity dispersion deteriorate seismic images hindering their interpretation and inversion. Removing the attenuation and dispersion of seismic records involves the use of inverse filters based on models that include the quality factor, $Q$, therefore, a robust method should be used to estimate $Q$ and provide reliable values to improve the image of the subsurface value. The matching pursuit decomposition (MPD) generates a frequency spectrum for each sample of the trace (instantaneous), where reliable $Q$ values are estimated without the inconveniences of spectral analysis with windows based on the Fourier and Gabor transforms. Since the phase component of the filter is inherently stable, compensation applies only to the amplitude. MATLAB algorithms that implement both the MPD and the stable inverse filter for removing the effects of attenuation and dispersion on pre and post-stack data were developed. The $Q$ value estimated on synthetic seismograms and used with an inverse filter allowed correcting the distortion of the wavelet by retrieving the amplitude and phase spectra. The methodology was applied to a pre-stacked marine seismic line PSTM increasing the signal-noise ratio and significantly improving the temporal resolution records.
\end{abstract}

Key words: Attenuation, dispersion, instantaneous spectrum, matching pursuit decomposition, inverse filter, quality factor. 


\section{Introducción}

Al viajar en un medio, las ondas sísmicas sufren la disminución de las amplitudes de sus armónicos de mayor frecuencia, lo que reduce el ancho de banda útil de los datos, la cual, además, se ve sujeta a distorsión debido a la diferencia de las velocidades de fase de sus armónicos. Estos fenómenos oscurecen las imágenes del subsuelo y dificultan su interpretación, haciendo inestable la inversión de las secciones sísmicas. Son muchos los enfoques que se han aplicado con éxito relativo para aumentar la resolución de tiempo mediante la inversión espectral (Castaño, et al., 2011; Smith, et al., 2008), o mediante el análisis de ampliación espectral (Kazemeini, et al., 2010). Por otro lado, el factor de calidad cuantifica la atenuación como el porcentaje de energía perdida en un ciclo con determinada frecuencia, por lo que se han desarrollado métodos para aplicar filtros inversos que usan un factor de calidad inverso a los datos sísmicos (Walls, et al., 2006), y cuyo desempeño ha sido comparado (Montana \& Margrave, 2005). El factor de calidad, que depende de la frecuencia, mide la capacidad de la roca para atenuar ondas sísmicas y por ello las diversas composiciones de las capas modifican la atenuación hasta esa profundidad. Por consiguiente, la detección de cambios en la atenuación permitiría establecer las propiedades líticas del subsuelo (Ramírez-Cruz, et al., 2005). El método de la relación espectral (Spectral Relaxation Method, SRM) es el más comúnmente utilizado para calcular el factor de atenuación por intervalo. En un comienzo se usó ampliamente para estimar la atenuación en registros de perfil sísmico vertical (Vertical Seismic Profile, VSP) acústicos $\mathrm{y}$, recientemente, en los registros de VSP de cizalla (Xu \& Stewart, 2006). Además, estas nuevas metodologías se han aplicado para el cálculo de la onda $\mathrm{P}$ y la atenuación de las ondas S (Qp y Qs) a partir de datos de registros de pozos estándar con para relacionarlas con la presencia de petróleo o gas natural (Wang, et al., 2009). Mediante su procesamiento se busca recuperar las amplitudes y las fases originales de las ondas, aplicando filtros que dependen de $Q$, por lo que su errónea estimación introduciría artefactos en las secciones sísmicas. Con este fin se han usado distintos métodos para estimar exactamente dicho factor $Q$, incluidas las medidas en registros de pozo, en núcleos de roca, en VSP y en datos sísmicos (Raikes \& White, 1984; Tonn, 1991). En el caso de las ondas de cizalla, el factor $Q$ se ha estimado mediante la adaptación del método de relación espectral usando un VSP cero-offset con valores confiables de $Q$ (Xu \& Stewart, 2006; Haase \& Stewart, 2006). La estimación precisa de $Q$ hace posible hacer análisis AVA/

\footnotetext{
*Correspondencia:

Luis Montes-Vides, lamontesv@unal.edu.co

Recibido: 20 de marzo de 2014

Aceptado: 4 de agosto de 2014
}

AVO confiables, lo que disminuye el riesgo exploratorio mediante una inversión sísmica que permite caracterizar el reservorio. En consecuencia, la estimación precisa de $Q$ es crucial antes de realizar una inversión, pero dado que la atenuación no está linealmente relacionada con la frecuencia (Kolsky, 1956), dicha estimación se convierte en una tarea compleja. Wang (2006) propuso un método inverso para eliminar la atenuación y la dispersión de los datos sísmicos sin amplificar la señal cuando esta se encuentra bajo el umbral del ruido y mejorar la relación señal-ruido. Este método se basa en una estimación precisa del valor de $Q$; sin embargo, la precisión y la exactitud alcanzadas dependen de la transformada que suministra el espectro sobre el cual se puede estimar $Q$ (Reine, et al., 2009). La transformada adaptable en tiempo y frecuencia, MPD, ha demostrado su eficiencia y solidez para suministrar espectros instantáneos precisos al usar un diccionario de ondículas mediante las cuales se puede representar la señal con exactitud (Wang, 2007), siendo las ondículas de Morlet las que mejor desempeño alcanzan en este cometido (Liu \& Marfurt, 2005). El espectro instantáneo $t$ - $\omega$ se transforma en un nuevo dominio $\chi$ en el cual $Q(\chi)$ se calcula con exactitud usando una regresión lineal. En este caso, se implementó un filtro $Q$ inverso estabilizado, el cual restablece los espectros de amplitud y de fase sin aumentar el ruido ambiental y mejora la relación entre señal y ruido de los datos. Se presenta aquí el resultado de la aplicación, tanto en datos sintéticos como en datos reales, de un filtro inverso estable que usa un valor de $Q$ estimado en un espectro instantáneo suministrado por la MPD, con lo que se logra una recuperación de los espectros de amplitud y de fase de la señal y se obtienen registros de mejor calidad. Los algoritmos que implementan la MPD y el filtro inverso fueron especialmente desarrollados para este proyecto.

\section{Fundamento teórico}

\section{Descomposición adaptable tiempo-frecuencia}

La técnica de selección adaptable (matching pursuit decomposition, MPD) es frecuentemente usada en el procesamiento sísmico para reconstruir señales mediante diccionarios de clases de funciones y hallar aquellas que forman parte de la señal. El diccionario $\left\{\Psi_{j}\right\}_{j=1}^{n}$ se construye con funciones previamente calculadas, las cuales se estiman y eligen al ajustar mejor la traza sísmica $x(t)$, reconstruyéndola por medio de su combinación lineal: $x(t)=\sum_{j=1}^{n} s_{j} \Psi_{j}$. Estas funciones reciben el nombre de átomos y tienen la siguiente forma compleja:

$$
\Psi_{j}=s^{-1 / 2} \Psi\left(t-t_{d} / s\right) \exp i f_{\mathrm{m}} t
$$

donde $i=\sqrt{-1}, f_{\mathrm{m}}$ es la frecuencia de modulación, $s$, el factor de escala y $t_{d}$, el tiempo de translación. Se seleccionó la ondícula de Morlet como función madre debido a que se ajusta mejor a las ondas sísmicas e incluye la atenuación y la dispersión 
cuantitativas (Sinha, et al., 2005). En cada iteración, el algoritmo MPD busca la ondícula que proporciona la mayor parte de la energía de la señal y la elimina. La energía de la señal se reduce de manera reiterada hasta alcanzar la energía umbral establecida previamente, en tanto que la energía restante se considera ruido. La figura 1S, http://www.raccefyn.co/index. php/raccefyn/article/downloadSuppFile/125/380, muestra el diagrama de flujo que implementa la MPD, el cual se codificó en MATLAB para el desarrollo del presente trabajo.

\section{Atenuación y dispersión sísmica}

Cuando una onda monocromática atraviesa un medio con velocidad de fase $c$, parte de su energía se disipa en forma de calor. El decaimiento de la señal por ciclo se mide mediante el factor de atenuación $\propto=Q^{-1}(\omega / 2 c)$, donde $Q$ es el factor de calidad del factor estimado como $\propto=27,3 Q^{-1}$ $\mathrm{dB}$ por longitud de onda $\lambda$. El modelo básico planteado por Kolsky (1956) para la atenuación y dispersión de una onda que se propaga en un medio, se representa mediante el factor $Q$; por su simplicidad, dicho modelo es el más utilizado en el procesamiento de datos sísmicos, ya que reproduce exactamente la dispersión de velocidades de fase dentro del ancho de banda de la frecuencia sísmica. La propagación de la onda en el medio viene dada por

$$
U(x, \omega)=U_{0}(\omega) \exp +i(k x-\omega t)
$$

donde $U_{0}(\omega)$ es la transformada de Fourier de la ondícula, $k$ es el número de onda, $x$, la distancia, $t$, el tiempo y $\omega$, la frecuencia. El número de onda $k$ se expresa como

$$
k(\omega)=\omega / v(\omega)-i \alpha(\omega)
$$

donde, según Kolsky (1956), la velocidad de fase $v(\omega)$ y el factor de atenuación $\alpha(\omega)$ están dados por

$$
\begin{gathered}
v(\omega)^{-1}=\left(1+\ln \left|\omega / \omega_{r}\right|^{-1 / \pi Q_{r}}\right) / v_{r} \\
\alpha(\omega)=|\omega| /\left(2 v_{r} Q_{r}\right)
\end{gathered}
$$

en las que $v_{r}$ es una velocidad de referencia aproximadamente igual a la velocidad de grupo y $Q_{r}$ es el factor de calidad a una frecuencia de referencia $\omega_{r}$. Para materiales que cumplan la suposición de atenuación lineal con la propagación, como es el caso de las rocas, la frecuencia de referencia $\omega_{r}$ no es nula, aunque sí es arbitrariamente pequeña comparada con la menor frecuencia contenida en la señal (Kolsky, 1956). Empero, el modelo de Kolsky no satisface rigurosamente el criterio de fase mínima y las relaciones de Kramers-Krönig. Para que el filtro inverso corrigiera plenamente la dispersión de velocidad, se propuso un modelo válido para frecuencias bajas (menores de $500 \mathrm{~Hz}$ ), en el cual la velocidad de fase es modelada mediante la ecuación (Wang \& Guo, 2004):

$$
v(\omega)^{-1}=\left(1+\ln \left|h\left(\omega / \omega_{r}\right)\right|^{-1 / \pi Q_{r}}\right) / v_{r}
$$

donde $h$ es una constante tal que el valor $h / \omega_{r}$ corresponde a la mayor frecuencia de la banda sísmica, y el factor de atenuación dado por la ecuación 5 se aproxima a:

$$
\alpha(\omega) \approx \frac{|\omega|}{2 v_{r} Q(\omega)}\left|\frac{\omega}{\omega_{h}}\right|^{-\gamma}
$$

en la que $\gamma=\left(\pi Q_{r}\right)^{-1} \mathrm{y}$, en consecuencia, la ecuación 3 se torna en

$$
k(\omega) \approx\left(1-\frac{i}{2 Q(\omega)}\right) \frac{|\omega|}{v_{h}}\left|\frac{\omega}{\omega_{h}}\right|^{-\gamma}
$$

$U(\omega, \tau+\Delta \tau)$ es la continuación del campo de onda $U(\omega, \tau)$ a través de la distancia $\Delta r$ en un intervalo de muestreo $\Delta \tau$, que, en el dominio de la frecuencia, se representa por:

$$
U(\omega, \tau+\Delta \tau)=U(\omega, \tau) \exp \left[\left(\frac{\omega}{\omega_{h}}\right)^{-\gamma} \frac{\omega \Delta \tau}{2 Q(\omega)}\right] \exp \left[i\left(\frac{\omega}{\omega_{h}}\right)^{-\gamma} \omega \Delta \tau\right]
$$

donde el primer exponencial modifica la amplitud y el segundo, la fase. La continuación del campo desde la superficie en $\tau_{0}=0$ hasta un tiempo $\tau$ estará dada por

$$
\begin{gathered}
U(\omega, \tau)=U(\omega, 0) \exp \left[\int_{0}^{\tau} \frac{\omega}{2 Q\left(\tau^{\prime}\right)}\left(\frac{\omega}{\omega_{h}}\right)^{-\gamma\left(\tau^{\prime}\right)} d \tau^{\prime}\right] \\
\exp \left[i \int_{0}^{\tau}\left(\frac{\omega}{\omega_{h}}\right)^{-\gamma\left(\tau^{\prime}\right)} \omega d \tau^{\prime}\right]
\end{gathered}
$$

y al denominar el primer exponencial como $\beta(\omega, \tau)^{-1}$, la ecuación se soluciona como problema inverso estabilizado, introduciendo $\Lambda(\omega, \tau)=\left(\beta(\omega, \tau)+\sigma^{2}\right) /\left(\beta^{2}(\omega, \tau)+\sigma^{2}\right)$, donde $\sigma^{2}$ es el factor estabilizador. La ecuación 10 se escribe ahora como

$$
\begin{aligned}
U(\omega, \tau)= & U(\omega, 0) \Lambda(\omega, \tau) \exp \\
& +i \int_{0}^{\tau}\left(\omega / \omega_{h}\right)^{-\gamma\left(\tau^{\prime}\right)} \omega d \tau^{\prime}
\end{aligned}
$$

La ecuación 11 se resuelve para cada frecuencia, y la integral de estas se expresa en la solución:

$$
\begin{aligned}
u(\tau)= & \frac{1}{\pi} \int_{0}^{\infty} U(\omega, 0) \Lambda(\omega, \tau) \\
& \exp \left[i \int_{0}^{\tau}\left(\frac{\omega}{\omega_{h}}\right)^{-\gamma\left(\tau^{\prime}\right)} \omega d \tau^{\prime}\right] d \omega
\end{aligned}
$$

\section{Aplicación en sismogramas sintéticos}

La descomposición espectral tiempo-frecuencia de una traza sísmica mediante el algoritmo MPD se muestra en el diagrama de flujo de la figura 1S. Este se inicia con la construcción de un diccionario de ondículas de Morlet, implementado como una matriz tridimensional que almacena las ondículas con los rangos de fase y frecuencia dominantes en la traza sísmica. Las ondículas del diccionario de Morlet se construyeron usando los rangos de valores de la tabla 1S, http://www.raccefyn.co/index.php/raccefyn/article/ downloadSuppFile/125/384, en la ecuación:

$$
M(t)=\exp \left(-\gamma f_{m}^{2} t^{2}\right) \exp \left(i 2 \pi f_{m} t+\Phi\right)
$$


El rango de tiempo garantiza la presencia de las ondículas de baja frecuencia, mientras que los rangos de frecuencia y de fase incluyen los valores presentes en el ancho de banda de una traza sísmica, y el parámetro $\gamma$ fija el número de ciclos en la ondícula de Morlet. Usando la transformada de Fourier de la ondícula de Morlet, $M(\omega)$, y el número de onda $k \omega$, definido en la ecuación 8, se construye el sismograma sintético $u(t)$ con la expresión:

$$
u(t)=\operatorname{Re}\left\{\frac{1}{\pi} \int_{0}^{\infty} M(\omega) \exp [i(\omega t-k r)] d \omega\right\}
$$

En un primer paso, se construyó una traza sintética sumando las siguientes convoluciones: la ondícula de Morlet de $20 \mathrm{~Hz}$ y fase cero, con los coeficientes de reflectividad 1 y -1 a 300 y $900 \mathrm{~ms}$; la ondícula de Morlet de $30 \mathrm{~Hz}$ y fase de $45^{\circ}$, con los coeficientes de reflectividad 1,1, 1 y 1 a 450, 930 y $1.200 \mathrm{~ms}$, y, finalmente, la ondícula de Morlet de $50 \mathrm{~Hz}$ y fase de $90^{\circ}$, con coeficientes de reflectividad 1 y 1 a 600 y $1.230 \mathrm{~ms}$. La figura 2SA, http:/www.raccefyn.co/index. php/raccefyn/article/downloadSuppFile/125/381, muestra la traza original, 2SB la traza generada con el Diccionario, 2SC el residuo o error entra las trazas anteriores y 2SD el espectro instantáneo $\omega-t$ de alta resolución. Cada impulso del perfil de reflectividad en la figura $2 \mathrm{SE}$ coincide con el espectro de cada ondícula en la figura 2SD, lo que permite determinar las bandas de frecuencias de cada ondícula y garantizar el buen desempeño del algoritmo MPD para obtener un espectro altamente confiable.

A continuación se creó un sismograma sintético de $2 \mathrm{~s}$ de longitud de registro usando la ecuación 15 en un modelo inelástico, con $Q=100$ constante, y reflectores cada 300 ms, de los cuales el más somero se situó a 100 ms y se analizó espectralmente mediante el algoritmo MPD, con un umbral para el residuo de energía de $0,1 \%$. Para observar la modificación de la amplitud y la fase en el cambio de forma de la ondícula, se usó la ondícula de Morlet fase cero de $30 \mathrm{~Hz}$ con $\gamma=10$, la cual tiene menos ondulaciones. La figura 3SA, http://www.raccefyn.co/index.php/raccefyn/ article/downloadSuppFile/125/382, muestra el sismograma generado, en donde se observa la distorsión que sufre la ondícula al disminuir su amplitud y cambiar su forma simétrica inicial de fase cero a una forma asimétrica a medida que avanza. En la figura 3SB, el espectro instantáneo de la traza evidencia la atenuación de los componentes de mayor frecuencia en profundidad. La máxima frecuencia de la ondícula disminuye de $100 \mathrm{~Hz}$ en el reflector somero a 55 en el más profundo. Debido a la ausencia de dispersión geométrica y a que los coeficientes de reflectividad son iguales o mayores a uno, el efecto observado es atribuible completamente a los fenómenos de atenuación y dispersión.

Mediante la sustitución $\chi=\omega \tau$, la ecuación de atenuación $A(\omega, \tau)=A_{0} \exp [-\omega, \tau / 2 Q(\omega)]$ cambia a la expresión lineal:

$$
\ln A^{2}(\omega, \tau)=\ln A_{0}^{2}-\omega \tau / Q(\omega)
$$

Al hacer la sustitución $\chi=\omega \tau$, la amplitud del espectro instantáneo pasará a depender solo de $\chi$ en lugar de $\omega$ y de $\tau$, resultado que cambia la ecuación 15 a la expresión lineal:

$$
\ln A^{2}(\chi)=\ln A_{0}^{2}-\frac{1}{Q} \chi
$$

$\mathrm{y}$ al denominar como $A_{0}=\left(\chi_{\alpha}\right)$ la amplitud en el punto referente $\chi_{\alpha}$, donde la ecuación 16 alcanza su máximo valor y decae linealmente con $\chi$, esta se reescribe como:

$$
\ln A^{2}(\chi)-\ln A^{2}\left(\chi_{a}\right)=-\left(\chi-\chi_{a}\right) / Q
$$

en tanto que la estimación de $Q$ se hace como la pendiente de una nube de puntos usando una regresión lineal:

$$
Q=-\left[\chi-\chi_{a}\right] /\left\{2 \log \left[A(\chi) / A\left(\chi_{a}\right)\right]\right\}
$$

La figura 3SB muestra la distribución de $\chi$ con curvas de nivel sobre el espectro instantáneo generado al aplicar el algoritmo MPD sobre la traza de la figura 3SA. Los nuevos valores de $A^{2}(\chi)$ se calculan sumando los valores del espectro instantáneo situados a lo largo de las curvas de $\chi=$ constante, $\mathrm{y}$ así se obtiene el nuevo espectro de energía, como se muestra en la figura 4SA, http://www.raccefyn.co/ index.php/raccefyn/article/downloadSuppFile/125/383, con su máximo valor en $\chi_{\alpha}=30$. En el espectro $A^{2}(\chi)$ se nota la ausencia de amplitudes alrededor de $\chi=60$ debido a la poca contribución de las amplitudes a lo largo de la curva $\chi=60$, como se ve en la figura $3 \mathrm{SB}$; de igual manera, se ve que los valores de $\chi$ superiores a 500 contribuyen muy poco a la energía total de la traza.

Mediante la ecuación 17, el espectro de la figura 4SA se transforma en el espectro de la figura 4SB, con valores negativos en la abscisa, correspondiendo el cero a $\chi=\chi_{\alpha}$ de máxima potencia, mientras que los otros valores se normalizan con este punto de referencia. El punto donde se quiebra la tendencia del espectro en la figura 4SB, $\chi=470$, indica el límite superior de la región donde se puede estimar $Q$. Mediante la regresión lineal de los datos y con la ecuación 18 , se obtuvo un valor estimado de $Q \approx 102$, con un error del $2 \%$, al compararlo con el $Q=100$ del modelo.

La función de atenuación normalizada $\overline{\mathrm{A}}(\chi)$, estimada en el espectro instantáneo, se suaviza con un filtro para retirar los valores atípicos y luego se determina la curva de ganancia normalizada:

$$
\Lambda_{\mathrm{d}}(\chi)=\left\{\begin{array}{cc}
1 & \chi \leq \chi_{\alpha} \\
{\left[\overline{\mathrm{A}}(\chi)+\sigma^{2}\right] /\left[\overline{\mathrm{A}}^{2}(\chi)+\sigma^{2}\right]} & \chi>\chi_{\alpha}
\end{array}\right\}
$$

El factor $\sigma^{2}$ estabiliza la parte del filtro inverso que compensa las amplitudes y que está ligada a la relación entre señala y ruido. Al ajustar los datos con límites de ganancia entre 10 y 100 dB, Wang (2008) determinó la relación empírica $\sigma^{2}=\exp -\left(0.23 G_{l i m}+1.63\right)$, donde $G_{l i m}$ es la ganancia 
A)

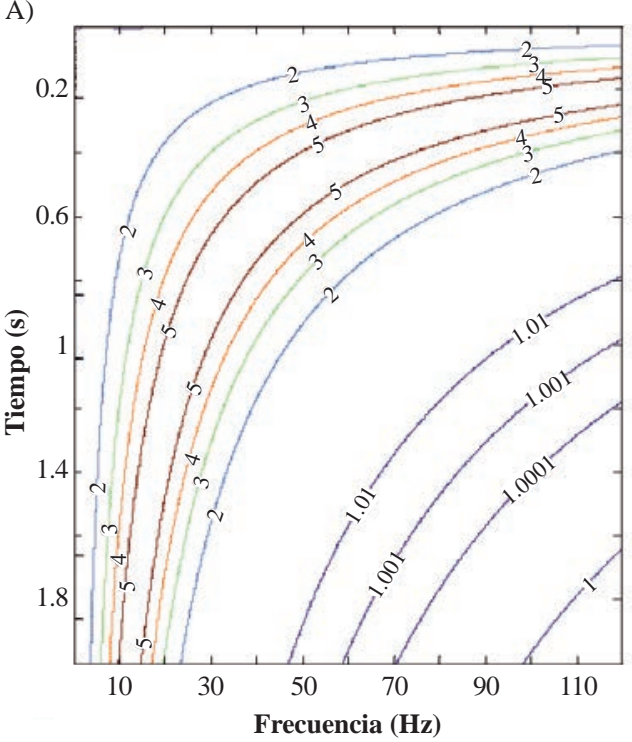

B)

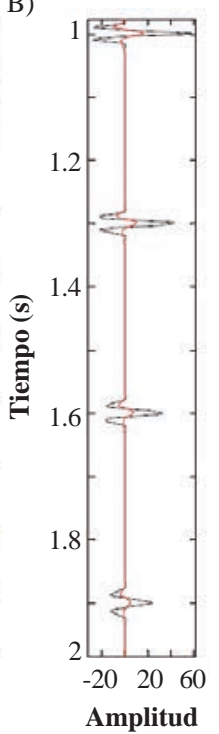

Figura 1. A) Coeficiente de compensación de amplitud $\Lambda(\omega, \tau)$ estimado para el modelo de atenuación constante, $Q=100$ y $\sigma^{2}=$ 0,01 . B) Intervalo de 1-2 segundos de los sismogramas sintéticos antes (rojo) y después (negro) de aplicar el filtro $Q$ inverso.

máxima esperada en $\mathrm{dB}$. Por otro lado, al definir la función de ganancia teórica $\Lambda(\omega, \tau)=\left(\beta(\omega, \tau)+\sigma^{2}\right) /\left(\beta^{2}(\omega, \tau)+\sigma^{2}\right)$ $\operatorname{con} \beta(\chi, Q)=\exp \left(-\frac{\chi}{2 Q}\right)$, se hace posible estimar el valor de $Q$ mediante la minimización de la función de costo $\Sigma_{\chi} \mid \Lambda_{d}$ $(\chi)-\Lambda(\chi, Q)$.

El filtro inverso descrito en la sección anterior se probó en el modelo sintético asumiendo un $\sigma^{2}=0,01$ y un límite de ganancia de $50 \mathrm{~dB}$, pues en los datos sintéticos se pueden asumir altos niveles de ganancia. La figura $1 \mathrm{~A}$ muestra el coeficiente de compensación de amplitud $\Lambda(\omega, \tau)$, donde la máxima compensación está alrededor de 5 , la cual disminuye con la frecuencia y el tiempo de viaje. La amplitud se compensa cuando la frecuencia es menor a $45 \mathrm{~Hz}$, mientras que para frecuencias mayores, la compensación $\Lambda$ es aproximadamente de 1, la cual corresponde a la información por debajo del nivel del ruido. Por otro lado, en las frecuencias bajas surgidas a lo largo del tiempo, la compensación también es baja, con valores de alrededor de 2 , así como es alta en las frecuencias altas presentes en la parte somera del registro, lo que garantiza que, en el peor escenario, el factor no compense las amplitudes, pero tampoco disminuya las originales. La figura 1B presenta el sismograma sintético antes (marrón) y después (negro) de retirar la atenuación y la dispersión mediante el filtro $Q$ inverso, limitado al último segundo para una mejor observación. Se aprecia la corrección de fase que contrarresta el efecto de dispersión de la velocidad, tornando a cero la fase de la ondícula, así como la restauración de la amplitud.

\section{Aplicación en los datos sísmicos preapilados}

El procedimiento de análisis espectral instantáneo con el filtro inverso $Q$ se hace traza a traza, y es aplicable a las trazas de un registro con punto medio común. Se utilizó un registro CDP (Common Depth Point Gather, CDP) de una sección sísmica marina cuyo relleno sedimentario consta, en general, de una secuencia de sedimentos carbonáticos y siliciclásticos del Paleógeno, seguida por una secuencia siliciclástica del Neógeno, registrada con intervalo de grupo de 100 m, 2 ms de rata de muestreo y una longitud de registro de $5 \mathrm{~s}$.

La figura 2A muestra una de las trazas del registro CDP, en tanto que la figura $2 \mathrm{~B}$ muestra el factor de compensación $\Lambda(\omega, \tau)$, con un comportamiento similar al observado en los datos sintéticos, pero con un ancho de banda menor, que alcanza una frecuencia máxima a $35 \mathrm{~Hz}$ aproximadamente y valores $\Lambda$ de 1,01 . La figura $2 \mathrm{C}$ muestra el espectro de energía en función de $\chi$, el cual corresponde a los primeros $2,5 \mathrm{~s}$ de registro después de la recuperación de las amplitudes por dispersión geométrica. La figura 2D muestra las curvas de ganancia controlada, tanto la teórica como la estimada a partir de los datos, con un buen ajuste entre ellas, lo que evidencia la confiabilidad del método con error de estimación, como se ve en la figura 2E. El punto mínimo,
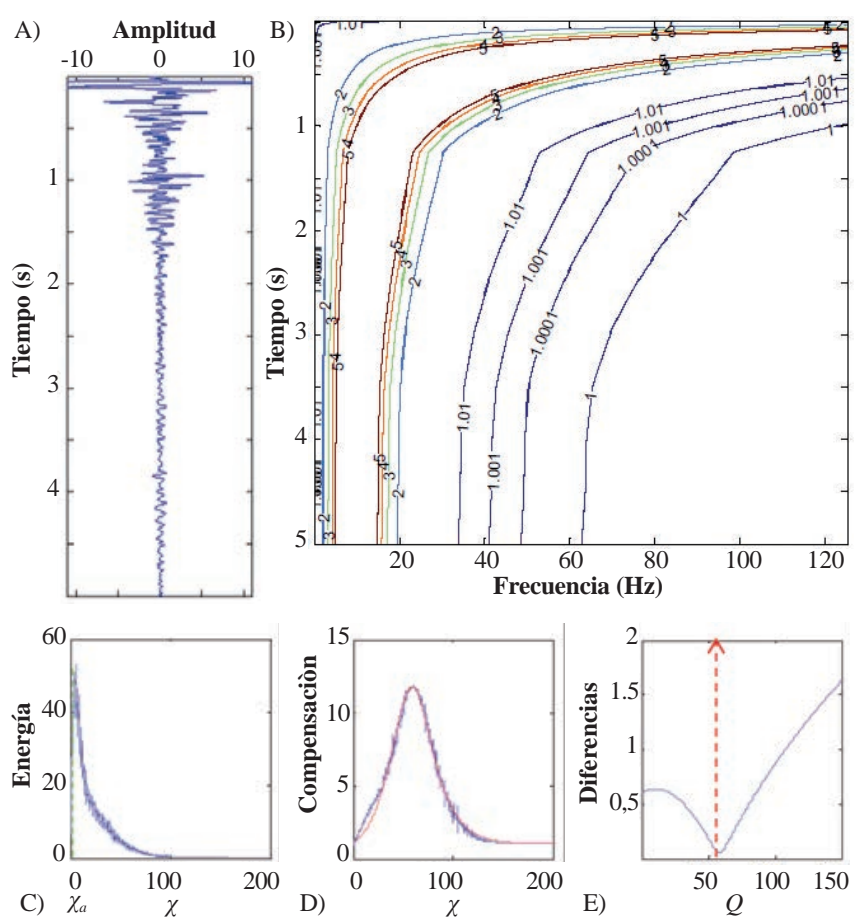

Figura 2. A) Traza sísmica del registro CDP, B) Coeficiente de compensación de amplitud $\Lambda(\omega, \tau)$ estimado en la traza, C) Espectro de energía de la traza en función de $\chi$, D) Curva de compensación teórica (línea suave) y curva estimada de los datos (línea rugosa), y E) Error en su estimación, donde el mínimo define el valor de $Q$. 
Tabla 1. Valores promedio de $Q$ estimados desde el tope de la sección sísmica y valores de $Q_{\text {int }}$ asociados a cada intervalo de tiempo

\begin{tabular}{cccc}
\hline Tiempo (s) & $\mathbf{Q}$ & Tiempo (s) & $\boldsymbol{Q}_{\text {int }}$ \\
\hline $0-1,25$ & 37,98 & $0-1,25$ & 37,98 \\
$0-1,5$ & 43,56 & $1,25-1,5$ & 164,13 \\
$0-2$ & 52,25 & $1,5-2$ & 130,13 \\
$0-2,5$ & 58,81 & $2-2,5$ & 118,14 \\
$0-3$ & 64,68 & $2,5-3$ & 129,12 \\
$0-3,5$ & 71,12 & $3-3,5$ & 176,65 \\
$0-4$ & 79,62 & $3,5-4$ & 487,31 \\
$0-4,5$ & 89,19 & $4-4,5$ & 2320,69 \\
$0-5$ & 97,84 & $4,5-5$ & 769,52 \\
\hline
\end{tabular}

indicado por una flecha, corresponde al valor de $Q$ estimado, que en este caso fue de 58,81. Los valores de $Q$ estimados para los diferentes intervalos de tiempo se consignan en la segunda columna de la tabla 1; dichos valores fueron usados posteriormente por el filtro $Q$ inverso para aplicarlos al registro. Con los $Q$ estimados, se calculó el valor $Q_{\text {int }}$ para cada intervalo de tiempo especificado en la tercera columna, el cual se consignó en la cuarta columna de la tabla 2.

La figura 3 muestra el registro CDP antes (A) y después (B) de aplicar el filtro $Q$ inverso. Se observa una mayor resolución en el registro filtrado, lo que permite una mejor definición de los eventos debido a la compresión de la ondícula, al mismo tiempo que se presenta una mayor continuidad lateral en

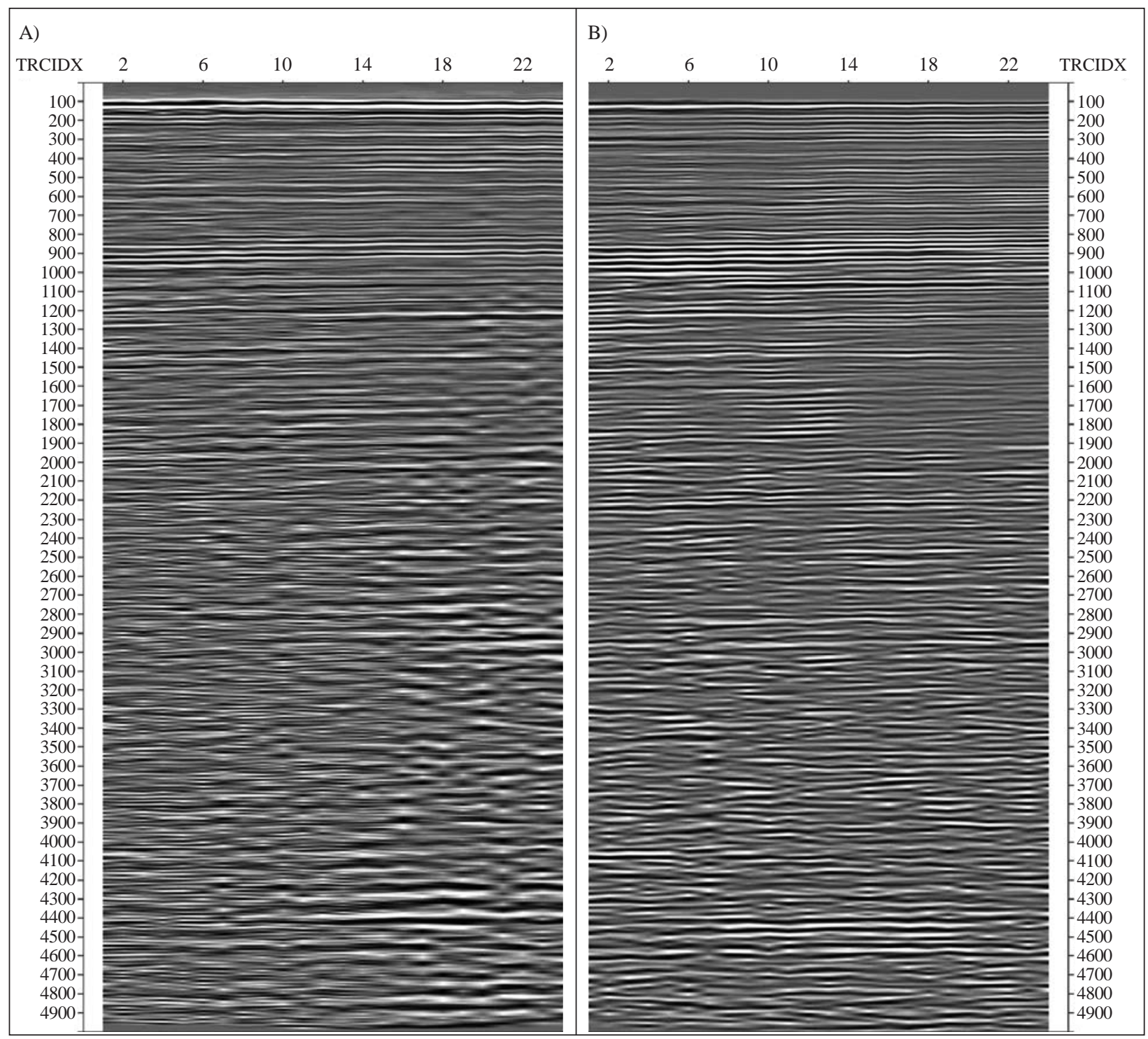

Figura 3. Registro CDP de la sección sísmica antes (A) y después (B) de aplicar el filtro $Q$ inverso. 


A)

Figura 4. Amplificación del intervalo somero de 400-820 s del registro CDP original (A) y después (B) del filtro Q inverso

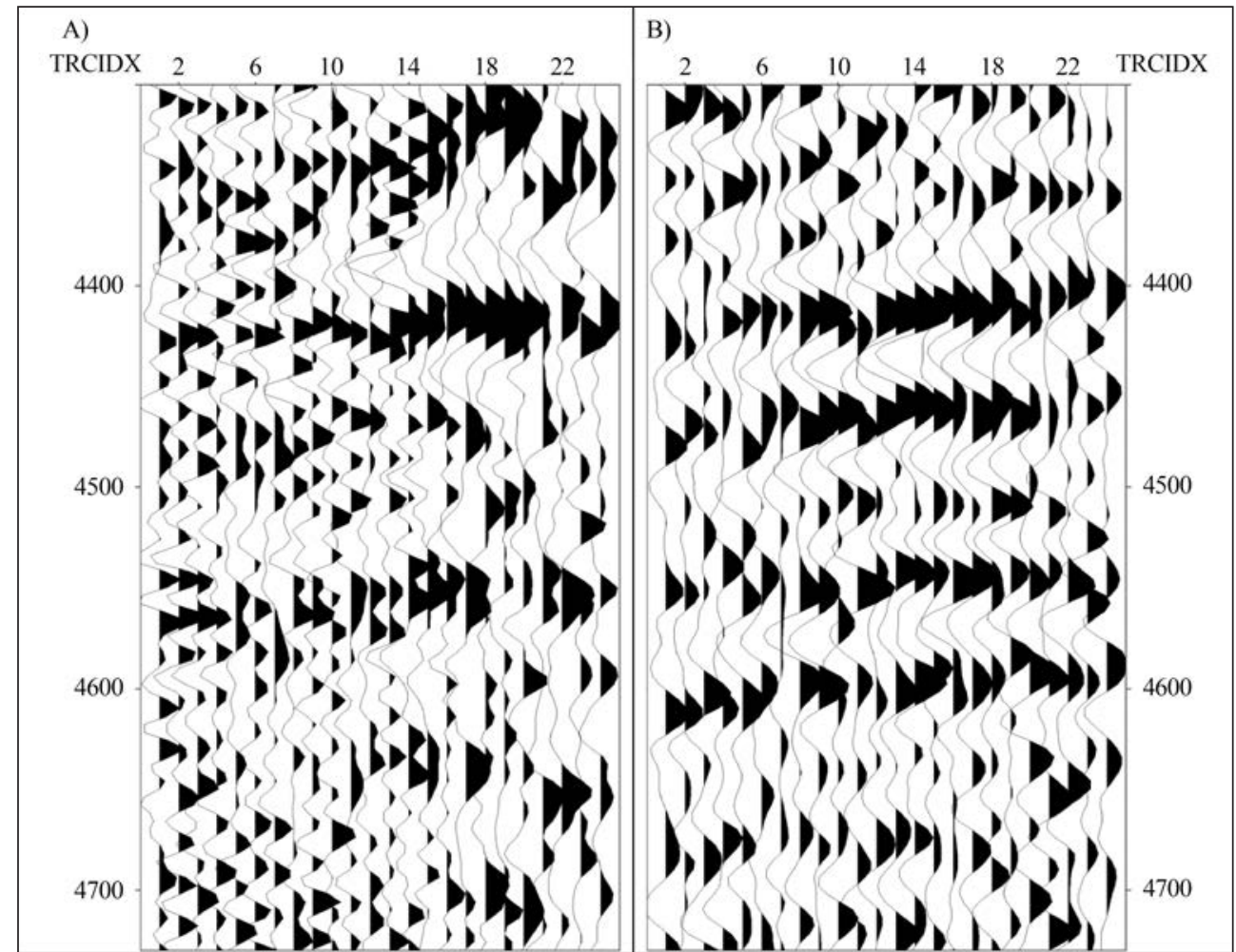

Figura 5. Amplificación del intervalo profundo de 4.300-4.720 s del registro CDP original (A) y después (B) de aplicar el filtro Q inverso 
algunos de ellos. La figura 4 amplifica el intervalo somero de 400 - 820 ms de la figura 3, donde, después de aplicar el filtro, se logra una mejor definición de los eventos entre los 550 y $650 \mathrm{~ms}$, así como una continuidad lateral ostensible. También se nota la aparición de un reflector alrededor de los $680 \mathrm{~ms}$, y de otro entre los 750 y $820 \mathrm{~ms}$. Se debe tener en cuenta que los datos preapilados tienen una relación entre señala y ruido menor que los datos apilados.

La figura 5 amplifica el intervalo profundo de 4.300 $4.720 \mathrm{~ms}$; en esta, algunas reflexiones, que lucen ruidosas y con fase no cero en el registro CDP original, logran una mejor definición mediante la compresión de las ondículas. Algunos eventos que no son claros en el registro original se evidencian después de aplicar el filtro, entre ellos, los eventos de amplitud positiva de 4.450 a $4.600 \mathrm{~ms}$. Aunque la información de preapilamiento contiene mayores niveles de ruido, el proceso de filtro inverso $Q$ logra reducirlos y mejorar la continuidad y el contenido de frecuencias de la sección, perfeccionando la calidad de las imágenes.

\section{Conclusiones}

Los filtros $Q$ se emplean rutinariamente para recuperar el ancho de banda de los datos sísmicos mediante la introducción de artefactos en las imágenes del subsuelo por distorsión de fase. Se implementó un filtro inverso que recupera el espectro de amplitud sin distorsionar la fase y mejora la relación entre señal y ruido mediante un factor de compensación y sin amplificar el ruido al final de los registros. Para garantizar la confiabilidad en los valores de $Q$ usados por el filtro, su estimación se lleva a cabo en un espectro instantáneo suministrado por un algoritmo que implementa la transformada adaptable en tiempofrecuencia, reduciendo la incertidumbre sobre este parámetro. El algoritmo que implementó el filtro $Q$ inverso se ensayó en datos sintéticos, lo que permitió revertir los fenómenos de atenuación y distorsión del sismograma sintético. Posteriormente, se aplicó a un registro CDP libre de dispersión geométrica al cual se le aplicó corrección dinámica. Se obtuvo así un registro con mayor resolución al comprimir la ondícula y una mejor continuidad lateral de los reflectores, tanto en la parte somera como en la parte profunda del registro. Las dos zonas amplificadas permitieron separar e identificar eventos asociados a los reflectores que mostraban una mejoría en su continuidad lateral. Los algoritmos que implementan la descomposición adaptable y el filtro $Q$ inverso fueron especialmente desarrollados para esta investigación.

\section{Información suplementaria}

Figura 1S. Diagrama de flujo del algoritmo MPD que realiza la descomposición adaptativa

Figura 2S. A) Sismograma original, B) Sismograma reconstruido mediante el diccionario, C) Error cometido al reconstruir el sismograma, D) Espectro instantáneo generado por el MPD y E) Perfil de reflectividad. Se observa la resolución de este espectro al compararlo con el perfil de reflectividad.
Tabla 1S. Rango de valores usados en la generación del diccionario

Figura 3S. A) Sismograma sintético generado, B) Espectro instantáneo del sismograma y superposición de las curvas de nivel de $\chi$.

Figura 4S. A) Espectro de energía en función de la variable $\chi$, y B) Representación del espectro según la ecuación 18, donde la pendiente es $\mathrm{Q}$.

\section{Agradecimientos}

Los autores agradecen a la Universidad Nacional de Colombia por apoyar y facilitar el desarrollo de esta investigación, así como a la Universidad de Houston, por suministrar los datos usados en este proyecto. Los resultados aquí presentados se lograron en el desarrollo de la tesis de Maestría en Geofísica del geólogo Álvaro Corrales.

\section{Bibliografía}

Castaño, K., Ojeda, G., Montes, L. 2011. Thin-layer detection using spectral inversion and a genetic algorithm. Earth Sci. Res. J. 15: 121-128.
Haase, A., Stewart, R. 2006. Intrinsic and apparent seismic attenuation in VSP data, SEG Technical Program Expanded Abstracts 2006: 3472-3476.

Kazemeini, S., Yang, C., Juhlin, C., Formel, S. 2010. Enhancing seismic data resolution using the prestack blueing technique: An example from the Ketzin CO2 injection site, Germany. Geophysics. 75: 101-110.

Kolsky, H. 1956. The propagation of stress pulses in viscoelastic solids. Philosophical Magazine. 1: 693-710.

Liu, J., Marfurt, K. 2005. Matching pursuit decomposition using Morlet wavelets. SEG Technical Program Expanded Abstracts 2005:786-789 
Montana, C., Margrave, G. 2005. Comparing three methods for inverse Q filtering. CSEG National Convention: 1-4.

Raikes, S., White, R. 1984. Measurements of earth attenuation from downhole and surface seismic recordings. Geophysical Prospecting. 32: 892-919.

Ramírez-Cruz, L., Vale-García, R., Urrutia, J. 2005. Enhanced oil production in a mature field assisted by spectral attenuation analysis. J. Geophys. Eng. 2: 48-53.

Reine, C., Van der Baan, M., Clark, R. 2009. The robustness of seismic attenuation measurements using fixed- and variable-window time-frequency transforms. Geophysics. 74: 123-135.

Sinha, S., Routh, P., Anno, P., Castagna, J. 2005. Spectral decomposition of seismic data with continuous-wavelet transform. Geophysics. 70: P19-P25.

Smith, M., Perry, G., Stein, J., Bertrand, A., Yu, G. 2008. Extending seismic bandwidth using the continuous wavelet transform. First break. 26: 97-102.
Tonn, R. 1991. The determination of seismic quality factor Q from VSP data: A comparison of different computational methods. Geophysical Prospecting. 39: 1-27.

Walls, J., Taner, M., Ude, R., Singleton, S., Derzhi, N., Mavko, G., Dvorkin, J. 2006. Novel use of P- and S-wave seismic attenuation for deep natural gas exploration and development. Final Report DE-FC26-04NT42243: 83-94.

Wang, Y., Guo, J. 2004. Modified Kolsky model for seismic attenuation and dispersion. J. Geophys. Eng. 1: 187-96.

Wang, Y. 2006. Inverse $Q$-filter for seismic resolution enhancement. Geophysics. 71: V651-V660.

Wang, Y. 2007. Seismic time-frequency spectral decomposition by matching pursuit. Geophysics. 72: V13-V20.

Wang, Y., Lu, J., Shi, Y., Yang, Ch. 2009. PS-wave Q estimation based on the P-wave Q values. J. Geophys. Eng. 6: 386-389.

Xu, C., Stewart, R. 2006. Seismic attenuation (Q) estimation from VSP data and Q P versus Vp/Vs. SEG Technical Program Expanded Abstracts 2006: 1938-1942. 